\title{
Pearls for Performing Laser-assisted Cataract Surgery
}

\author{
Elizabeth Yeu
}

Eastern Virginia Medical School, Norfolk, Virgnia, US; Virginia Eye Consultants, Norfolk, Virgnia, US

DOI: https://doi.org/10.17925/USOR.2016.09.02.78

aser assisted cataract surgery (LACS) is becoming more widely utilized, and comprised 10\% of cataract surgeries performed in the US in 2015 (Market Scope data). There are subtle differences from manual cataract surgery that can be challenging at first to get accustomed to, but with experience comes greater efficiencies that can be gained with the individual steps of phacoemulsification. LACS can provide the welcomed benefits of greater accuracy to low astigmatism correction, a more centered and standardized capsular openings, and mitigate certain difficulties associated with dense or otherwise complex cataracts.

\section{Keywords}

Laser assisted cataract surgery, femtosecond laser assisted cataract surgery, clinical pearls for laser cataract surgery

Disclosure: Elizabeth Yeu is a consultant to Alcon and Abbott. No funding was received in the publication of this article. This article is a short opinion piece and has not been submitted to external peer reviewers.

Open Access: This article is published under the Creative Commons Attribution Noncommercial License, which permits any noncommercial use, distribution, adaptation, and reproduction provided the original author(s) and source are given appropriate credit.

Received: August 4, 2016

Published Online: September 26, 2016

Citation: US Ophthalmic Review, 2016;9(2):78-9

Corresponding Author: Elizabeth Yeu, Virginia Eye Consultants, 241 Corporate Blvd, Norfolk, VA 23502, US. E: eyeulin@gmail.com
Laser-assisted cataract surgery has been a welcomed addition to my surgical arsenal for several reasons, including the greater refractive predictability provided by the standardized and wellcentered capsulorhexis, the increased accuracy of my relaxing keratotomies, and for the improved therapeutic efficiencies afforded in management of more complex cataracts. Overall, I think the learning curve is fairly steep for the experienced cataract surgeon, and there are certain nuances to the different steps of surgery, but femtosecond laser assistance lead to less stressful and more efficient cataract surgeries.

\section{Docking}

Each laser system has a unique docking system, whether it be a single piece patient interface (PI) seen with the LenS $x^{\circledR}$ SoftFit ${ }^{T M}$ (Alcon, Fort Worth, Texas) or a two-part docking PI that is seen with the Catalys ${ }^{\circledR}$ (Abbott Laboratories, Illinois, US) and the LENSAR ${ }^{\text {TM }}$, (LENSAR LLC, Orlando, Florida). Each has each own benefits and shortcomings, but in my experience, docking with even scleral show circumferentially between the limbus and the docked PI is essential. This helps to promote a flat dock, with less incidence of tilt during the laser treatment. A tilted eye during the treatment can lead to an incomplete capsulorhexis formation or the parallax can result in corneal wounds which are too anterior or posterior in location.

\section{Laser-assisted keratotomies}

Astigmatism can be more accurately treated because of the standardization of certain parameters Whereas the optical zone and depth are quite variable from patient to patient with manual limbal relaxing incisions, I almost always use an optical zone of $8.5 \mathrm{~mm}$ and $80 \%$ depth for my arcuate incision pattern. I commonly used single and paired incisions, but limit the incision length individually to be no larger than 40 degrees each. Larger than 40 degree arcs can lead to greater irregularity from contraction and gaping of the laser-created incision, and unpredictable outcomes. Thus, peripheral astigmatic keratotomies predictably and more accurately reduce 1 to 1.25 diopters of astigmatism. Attempting to correct any more can lead to greater refractive surprises, ocular surface irritation and dry eye disease.

\section{Hydrodissection}

Early on, this was the most daunting step for me because of the fear of compromising the posterior capsule from an aggressive hydrodissection. This certainly can happen because of the posteriorlytrapped air bubble that is created by the laser-assisted lens softening step. In my experience, the gas bubble is larger when creating a more limited treatment, like a single cross-hair chop pattern, because there are less fenestrated areas within the lens for the gas to pass through. I generally use a fragmentation or "waffle" pattern, and have appreciated that there is less gas trapped behind the lens nucleus. The technique of the hydrodissection should not change, but rather perform it a little slower 
and with less force than utilized in manual cases until experience leads to greater confidence. The gas bubble can actually be used to your advantage by propogating a combined hydro- and pneumodissection, particularly if the gas bubble is located more peripherally in location. For example, if the gas bubble appears towards the left side within the surgeon's view, starting the hydrodissection from the right side can direct the fluid around the lens and push the gas bubble anteriorly during the hydrodissection. If the gas does not budge, and there is concern about any further hydrodissection, proceed with the phacoemulsification in order to split the nucleus down the center. The gas bubble will more readily come forward through the center, and the hydrodissection step can be repeated with greater ease.

\section{Nuclear disassembly}

In general, this step is easier with the laser patterns. Each surgeon will customize a pattern that works well for them. "More" definitely does not necessarily lead to "better" during this step. More specifically, creating small, numerous fragmentation cubes can make it more difficult to purchase pieces because the individual cubes will release instead of the actual quadrant itself, particularly for softer lenses. Additionally, smaller cubes are the perfect size to sneak into the sulcus or zonules, and a greater incidence of retained lens fragments can occur as a result. Some lasers, such as the LENSAR, will automatically assign a grade based on the density of the lens, and customized lens patterns can be programmed for each specific grade or density. Other lasers do not have such automation. As a general rule of thumb, patients who are younger than 65 years of age do well with conservative patterns, and a single 4 quadrant chop pattern (which appears like a cross hair), will suffice well to help split the softer, often gummy lens. My patients between ages 65 and 75 will undergo a lens fragmentation pattern of 500 micron cubes. Those who are older than 75 years of age, of with known mature lenses, will undergo a lens fragmentation pattern with 300 micron cubes.

\section{Cortical removal}

The clean-up of the cortical fibers can be surprisingly challenging, particularly because this is often one of the easier steps in manual cataract surgery. Whereas in manual phacoeulsification, the rhexis does not disturb the cortex, laser capsulotomies actually create a sharp delineation of the cortex since the laser fires into this area during the capsulotomy creation. This results in a lack of any fimbriated cortex for the aspiration port to grab onto. In order to remove the cortex more readily, I find it most helpful to go out towards the equator where the cortex is and gently aspirate and sweep from the periphery towards the center. The sub-incisional cortex can be quite difficult with co-axial I/A, and this is where a bi-manual I/A approach can be easier and more efficient. $\square$ 